\title{
Research Article \\ Surface Effects on the Properties of Screw Dislocation in Nanofilms
}

\author{
Lili Liu, ${ }^{1,2}$ Zhenya Meng, ${ }^{2}$ Gang Xu, ${ }^{1}$ Chenglin He, ${ }^{1}$ Xiaozhi Wu, ${ }^{2,3}$ and Rui Wang ${ }^{2}$ \\ ${ }^{1}$ Department of Physics, Chongqing Three Gorges University, Chongqing 404100, China \\ ${ }^{2}$ Institute for Structure and Function, Chongqing University, Chongqing 401331, China \\ ${ }^{3}$ College of Materials Science and Engineering, Chongqing University, Chongqing 400044, China \\ Correspondence should be addressed to Xiaozhi Wu; xiaozhiwu@cqu.edu.cn
}

Received 13 November 2016; Accepted 16 January 2017; Published 5 February 2017

Academic Editor: Santiago Garcia-Granda

Copyright (c) 2017 Lili Liu et al. This is an open access article distributed under the Creative Commons Attribution License, which permits unrestricted use, distribution, and reproduction in any medium, provided the original work is properly cited.

The image dislocation method is used to construct the governing equation of dislocations in nanofilms. The classical Peierls-Nabarro equation can be recovered when the thickness of nanofilm is taken to be infinite. In order to determine the core width and Peierls stress of dislocations, the unstable stacking fault energies of $\mathrm{Al}$ and $\mathrm{Cu}$ nanofilms are calculated via the first-principle methods. It is found that surface effect can increase the Peierls stresses of screw dislocations in $\mathrm{Al}$ and $\mathrm{Cu}$ nanofilms.

\section{Introduction}

Thin film materials are of great importance in modern technology. They have a wide range of applications in engineering systems, such as highly integrated electronic circuits and microelectromechanical devices. It is widely accepted that defects such as dislocations play a key role in determining the mechanical properties of materials [1]. The classical PeierlsNabarro (P-N) dislocation model has been the most widely used model of dislocations in bulk materials [2,3]. Although powerful in its simplicity, it cannot deal with the dislocations in nanofilm with free surfaces $[4,5]$. Accounting for the free surface on the physical properties of dislocations in nanofilm is important, as the ratio of surface to volume is high. The effects of free surface on the dislocation properties cannot be neglected anymore; the dislocation behavior in thin films is different from that in bulk materials [6, 7]. Recently, Lee and $\mathrm{Li}$ constructed a half-space P-N model for dislocations near a free surface and they claimed that the mobility of the dislocation will be increased near the free surface $[8,9]$. Cheng et al. investigated Peierls stress of a screw dislocation below a free surface via a self-consistent semidiscrete variational P-N model using image dislocation method [10]. It is found that the free surface increases the Peierls stress and in consistence with the results obtained by molecular dynamics simulation
$[11,12]$. In this letter, the image dislocation method is used to investigate the dislocation core structure and Peierls stress in nanofilm with double free surfaces. The image dislocations are introduced to satisfy the free surface boundary condition; namely, the stress field acting on the surface should vanish [13]. The governing equation for dislocations in nanofilm is constructed according to the procedure for P-N equation provided by Joós et al. [14, 15]. Furthermore, the unstable stacking fault (USF) energies of $\mathrm{Al}$ and $\mathrm{Cu}$ nanofilms are also calculated by first-principle methods to discuss the effect of surface [16].

\section{Dislocations Properties in Nanofilms}

To facilitate the presentation we adopt the following conventions in all that follows (see Figure 1). In a Cartesian set of coordinates $x y z$, the $x o z$ plane is the slip plane and $y$-axis is normal to the slip plane. The $z$-axis is the direction of the screw dislocation and the direction of the Burgers vector. In an isotropic crystal and elastic continuum theory, the screw dislocation in bulk material produces a stress field $\sigma_{y z}$ along its Burgers vector [1]

$$
\sigma_{y z}=\frac{\mu b}{2 \pi} \frac{x}{x^{2}+y^{2}} .
$$




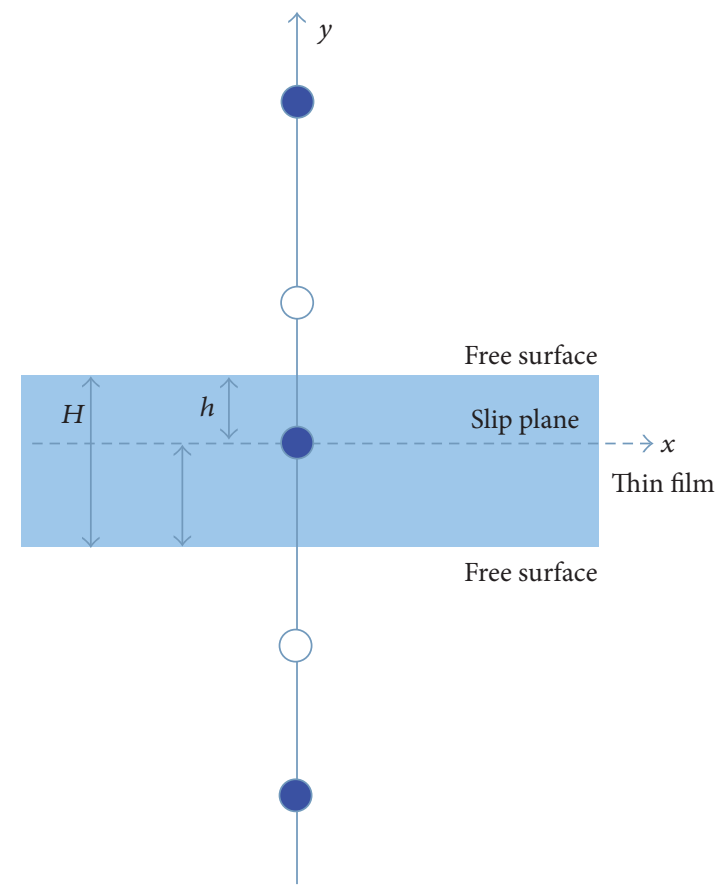

Right-handed

Left-handed

FIGURE 1: An array of imaginary dislocations of the original screw dislocation in the thin film. The slip plane of the original screw dislocation is parallel to the surfaces of the thin film and the effects of the surfaces can be described by the array imaginary dislocations. $H$ is the thickness of nanofilms, and $h \mid(H-h)$ shows the position of slip plane.

$\mu$ is the shear modulus. In order to take into account the surface effect on the screw dislocation properties, a screw dislocation with thickness $H$ at a distance $h$ below the upper free surface is considered in a nanofilm (see Figure 1). In the typical way to count for the image effect, the two image screw dislocations with the opposite Burgers vector $b^{\prime}=-b$ are distributed along a horizontal line at a distance $h$ above the upper free surface and $(H-h)$ below the lower free surface. These two image dislocations superpose new stresses which are equal and opposite to the already existing stresses on the upper and lower surfaces, respectively. However, the above (below) image screw dislocation leaves a residual stress acting on the lower (upper) free surface, so that an additional image is required, which in turn requires another image and so on. The result is an infinite set of image screw dislocations. The stress field of original screw dislocation in thin film combined with the surface effect can be equally produced by this infinite set of screw dislocations. Then, the stress field on the $y=0$ plane can be written as

$$
\begin{aligned}
\sigma_{y z}= & \frac{\mu b}{2 \pi} \frac{1}{x}+\frac{\mu b^{\prime}}{2 \pi} \frac{x}{x^{2}+(2 h)^{2}}+\frac{\mu b^{\prime}}{2 \pi} \frac{x}{x^{2}+(2 H-2 h)^{2}} \\
& +\frac{\mu b}{2 \pi} \frac{x}{x^{2}+(2 H)^{2}}+\frac{\mu b}{2 \pi} \frac{x}{x^{2}+(2 H)^{2}} \\
& +\frac{\mu b^{\prime}}{2 \pi} \frac{x}{x^{2}+(2 h)^{2}}+\frac{\mu b^{\prime}}{2 \pi} \frac{x}{x^{2}+(2 H-2 h)^{2}}
\end{aligned}
$$

$$
\begin{aligned}
& +\frac{\mu b}{2 \pi} \frac{x}{x^{2}+(4 H)^{2}}+\frac{\mu b}{2 \pi} \frac{x}{x^{2}+(4 H)^{2}} \\
& +\frac{\mu b^{\prime}}{2 \pi} \frac{x}{x^{2}+(4 H+2 h)^{2}}+\frac{\mu b^{\prime}}{2 \pi} \frac{x}{x^{2}+(6 H-2 h)^{2}} \\
& +\cdots
\end{aligned}
$$

It is helpful to rearrange the terms

$$
\begin{aligned}
& \sigma_{y z} \\
& =\frac{\mu b}{2 \pi}\left[\sum_{n=-\infty}^{+\infty} \frac{x}{x^{2}+(2 n H)^{2}}-\sum_{n=-\infty}^{+\infty} \frac{x}{x^{2}+(2 n H+2 h)^{2}}\right] .
\end{aligned}
$$

Using identities,

$$
\begin{gathered}
\sum_{n=-\infty}^{+\infty} \frac{1}{q^{2}+n^{2}}=\frac{\pi}{q} \operatorname{coth} \pi q \\
\sum_{n=-\infty}^{+\infty} \frac{1}{q^{2}+(n+p)^{2}}=\frac{\pi}{q} \frac{\sinh 2 \pi q}{\cosh 2 \pi q-\cos 2 \pi p}
\end{gathered}
$$

One obtains

$$
\begin{aligned}
& \sigma_{y z} \\
& \quad=\frac{\mu b}{4 H}\left[\operatorname{coth} \frac{\pi x}{2 H}-\frac{\sinh (\pi x / H)}{\cosh (\pi x / H)-\cos (2 \pi h / H)}\right] .
\end{aligned}
$$


In the above equation $b^{\prime}=-b$ is used. It is interesting to find that $\sigma_{y z}=0$ when $h=0$. The first-term of (3) represents the shear stress on $y=0$ plane of array right-handed screw dislocations siting $0, \pm 2 H, \pm 4 H, \ldots$. The second-term of (3) represents the shear stress on $y=0$ plane of an array left-handed screw dislocations siting $2 h, 2 H-2 h, 2 H+2 h, \ldots .$. It is also easy to verify that $\sigma_{y z}=0$ on $y=h$ and $y=-(H-h)$ planes; that is to say the free surface boundary condition is satisfied.

Like in the P-N model, an infinitesimal distribution of Burgers vector is introduced $\mathrm{d} b=\rho(x) \mathrm{d} x$ with $\rho(x)=$ $\mathrm{d} u(x) / \mathrm{d} x[14,15]$. The governing equation for the screw dislocation in thin film can be obtained by

$$
\frac{\mu}{4 H} \int_{-\infty}^{+\infty}\left[\operatorname{coth} \frac{\pi\left(x-x^{\prime}\right)}{2 H}-\frac{\sinh \left(\pi\left(x-x^{\prime}\right) / H\right)}{\cosh \left(\pi\left(x-x^{\prime}\right) / H\right)-\cos (2 \pi h / H)}\right] \frac{\mathrm{d} u\left(x^{\prime}\right)}{\mathrm{d} x^{\prime}} \mathrm{d} x^{\prime}=f(u) .
$$

The restoring force $f(u)$ can be obtained from the gradient of the generalized stacking fault (GSF) energy $\gamma_{u}$, which can be obtained from the first-principle calculation [16]. The USF energy, the maximum of the GSF energy, is the key role to control the properties of dislocation, such as the dislocation mobility and nucleation [17]. Therefore, the form of sinusoidal restoring force used here can be given as follows:

$$
\gamma(u)=\frac{\gamma_{\mathrm{us}}}{2}\left(1-\cos \frac{2 \pi u}{b}\right)
$$

with $\gamma_{\text {us }}$ being the USF energy. Then, we have the restoring force

$$
f(u)=\frac{\partial \gamma(u)}{\partial u}=\frac{\pi \gamma_{\mathrm{us}}}{b} \sin \frac{2 \pi u}{b} .
$$

There is a little difference comparing with the original sinusoidal restoring force $(\mu b / 2 \pi d) \sin (2 \pi u / b)$ used in the original P-N model. The maximum restoring force $\mu b / 2 \pi d$ is substituted by $\pi \gamma_{\mathrm{us}} / b$ for actual materials.

Dislocation equations can be deduced from (6) in two limitations: (i) dislocation in semi-infinite material with seldom surface $(H \rightarrow \infty)$, (ii) dislocation in bulk material without surface $(H \rightarrow \infty$ and $h \rightarrow \infty)$, and (iii) dislocation in the middle of the nanofilms $(h=H / 2)$.

(i) When $H \rightarrow \infty$, the first-term of (6) becomes the usual P-N integral for an isolated screw dislocation, and the second-term of (6) becomes the integral for imaginary screw dislocation at a distance $h$ below the free surface. Hence, the dislocation equation can easily yield

$$
\begin{aligned}
& \frac{\mu}{2 \pi} \int_{-\infty}^{+\infty}\left[\frac{1}{x-x^{\prime}}-\frac{x-x^{\prime}}{\left(x-x^{\prime}\right)^{2}+(2 h)^{2}}\right] \frac{\mathrm{d} u\left(x^{\prime}\right)}{\mathrm{d} x^{\prime}} \mathrm{d} x^{\prime} \\
& \quad=f(u) .
\end{aligned}
$$

This equation is the same as previous dislocation equation obtained by Cheng et al. for semi-infinite material with seldom free surface [10].

(ii) When $H \rightarrow \infty$ and $h \rightarrow \infty$, one obtains the classical $\mathrm{P}-\mathrm{N}$ dislocation equation in bulk

$$
\frac{\mu}{2 \pi} \int_{-\infty}^{+\infty} \frac{1}{x-x^{\prime}} \frac{\mathrm{d} u\left(x^{\prime}\right)}{\mathrm{d} x^{\prime}} \mathrm{d} x^{\prime}=f(u)
$$

(iii) When $H=2 h$, we can get dislocation equation in the middle of the nanofilms

$$
\frac{\mu}{2 H} \int_{-\infty}^{+\infty} \frac{1}{\sinh \left(\pi\left(x-x^{\prime}\right) / H\right)} \frac{\mathrm{d} u\left(x^{\prime}\right)}{\mathrm{d} x^{\prime}} \mathrm{d} x^{\prime}=f(u) .
$$

In this letter, for the dislocation equation (6) the dislocation solution can be written as

$$
u(x)=\frac{b}{\pi} \arctan \left[\frac{H}{\pi \zeta} \sinh \left(\frac{\pi x}{H}\right)\right]+\frac{b}{2}
$$

with $\zeta=\eta \zeta_{0}$ and $\zeta_{0}=d / 2 . \eta$ is the core width parameter of screw dislocation in thin film. Equation (12) tends to the original Peierls solution $u(x)=(b / \pi) \arctan \left(x / \zeta_{0}\right)+b / 2$, when $H \rightarrow \infty$ [14]. It is interesting to find that (12) is the suitable approximation solution of these equations with $\eta \simeq 1$ for original sinusoidal force law $(\mu b / 2 \pi d) \sin (2 \pi u / b)$ in simple cubic lattice. In this letter, the suitable approximation can be interpreted as the relative error from the sinusoidal law of force, and the relative error in the core region decreases with thickness of nanofilm. This also means that the core width of screw dislocation is nearly independent on the thickness of nanofilm. The classic Peierls solution is also used to solve these equations, but asymptotic behavior of the stress and restoring force is not consistent far from dislocation.

Generally, a narrow dislocation core implies a relatively high Peierls stress. In this letter, the Peierls stresses are evaluated through the calculation of the misfit energy $E$ taken as the sum of the local misfit energies between pairs of atomic planes [14]

$$
E\left(x_{0}\right)=\sum_{n=-\infty}^{+\infty} \gamma\left[u\left(n b-x_{0}\right)\right]
$$

The minimum stress required to overcome the energy barrier $E\left(x_{0}\right)$ is then defined as the Peierls stress $\sigma_{p}$

$$
\sigma_{p}=\max \left\{\frac{\mathrm{d} E\left(x_{0}\right)}{\mathrm{d} x_{0}}\right\}
$$

Surface effect contains two parts: one introduces the stress field of image dislocation that changes the form of dislocation equation and the other results in the changing of GSF energy. Thus, it means that the primary role to dislocation properties 


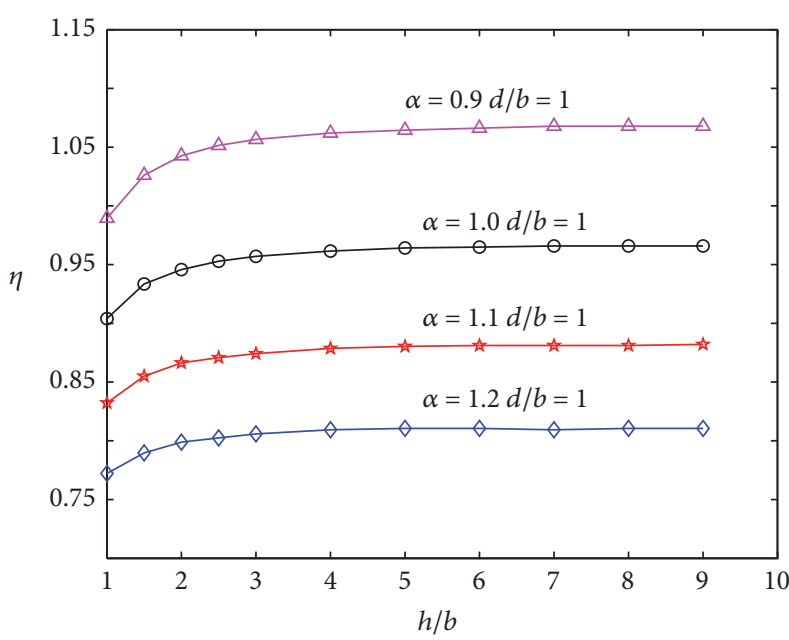

(a) Semi-infinite Crystal

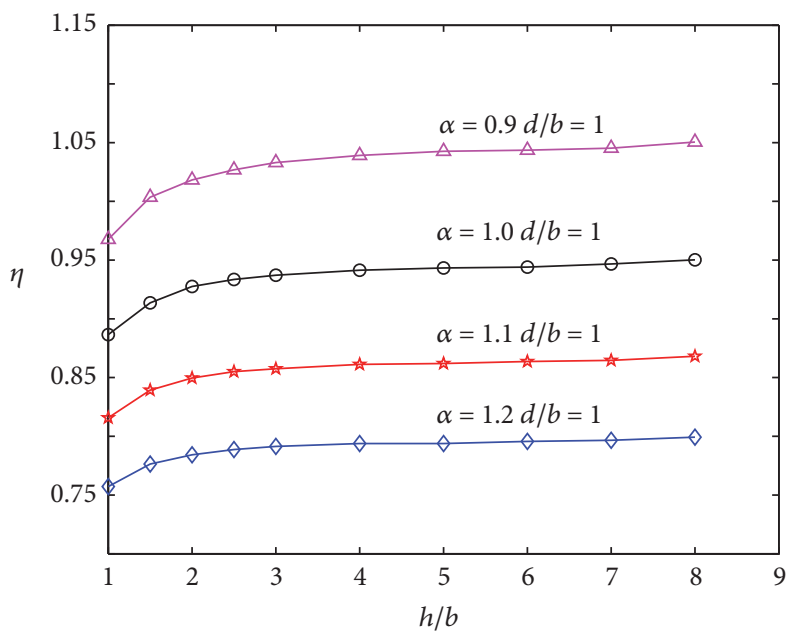

(c) $H / b=18$

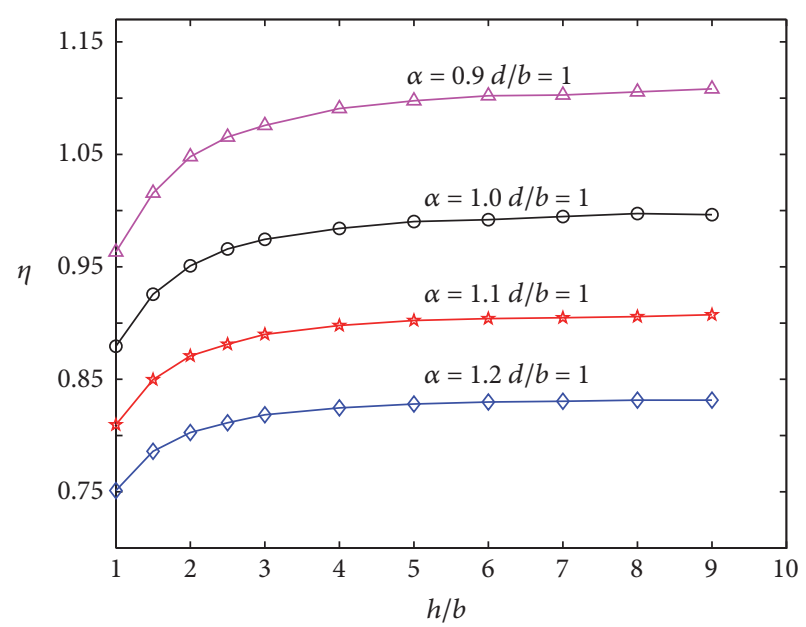

(b) $h=H / 2$

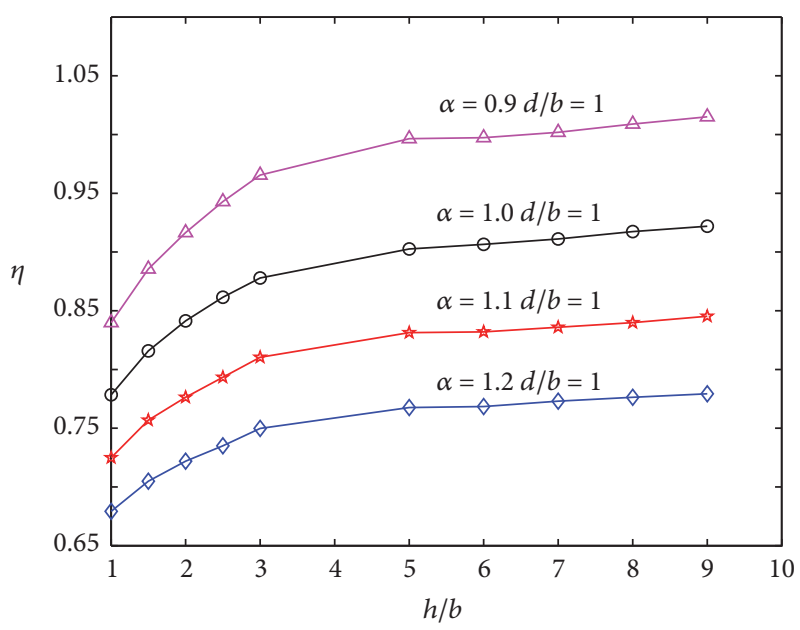

(d) $H / b=h / b+4$

FIGURE 2: Variation of core structure parameter $\eta$ with respect to the dislocation's distance to the surface for various normalized unstable stacking fault energy for screw dislocations.

is originated from the GSF energy and the effect of stress field of image dislocations is small.

To research how the core width $(\xi)$ of screw dislocation changes with the interplanar atomistic spacing $(d / b)$, the core structure parameters $(\eta)$ are plotted with respect to its distances to the free surface $(h / b)$ for different $\alpha$ and $d / b$ in Figures 2 and 3 , respectively. In our study, $\gamma_{\mathrm{us}}=\alpha \cdot \mu b^{2} / 2 \pi^{2} d$ instead of $\gamma_{\text {us }}=\mu b^{2} / 2 \pi^{2} d$, with $\alpha$ in the range of $0.8 \sim 1.2$. The range of $d / b$ studied is $0.7 \sim 1.4$ in real crystals, and the range for $h / b$ studied is $1 \sim 9$, because the surface effect becomes less significant only when the dislocation is more than 9 layers of atoms away from the free surface. This result is consistent with Cheng et al. [10] in semi-infinite crystal (see Figure 2(a)). Unlike Cheng et al. [10], we are more concerned with surface effects on the properties of screw dislocation in nanofilms. The core structure parameter $(\eta)$ in nanofilms is influenced by $h$ as well as the nanofilms thickness $(H)$. Therefore, three kinds of models are constructed in order to investigate the interaction between the dislocation and double free surfaces: (1) screw dislocation in the middle $(h=H / 2)$ of the nanofilms; (2) nanofilms thickness $H / b=18$; (3) the height of the distance below the surface $(H-h) / b=4$. It is found that, similar to the results for the semi-infinite crystal, the core structure parameter $(\eta)$ decreases when the screw dislocation in nanofilms gets closer to the surface with the same $\alpha$ and $d / b$; it means that screw dislocation near the surface causes a narrower dislocation core (Figures 2(b)-2(d)). The increase of $\alpha$ will also lead to the reduction of the core structure parameter $(\eta)$ with the same $h / b$ and $d / b$, but the core structure parameter $(\eta)$ increases with increasing $d / b$ with the same $h / b$ and $\alpha$ (see Figure 3).

In order to investigate the effect of free surface on the Peierls stress, the variation of $\sigma_{p} / \sigma_{p}^{0}$ of a screw dislocation with respect to its distance $(h)$ to the free surface for different $\alpha$ and $d / b$ is shown in Figures 4 and 5 , respectively, where $\sigma_{p} / \sigma_{p}^{0}$ is the amplification factor for the Peierls stress. As 


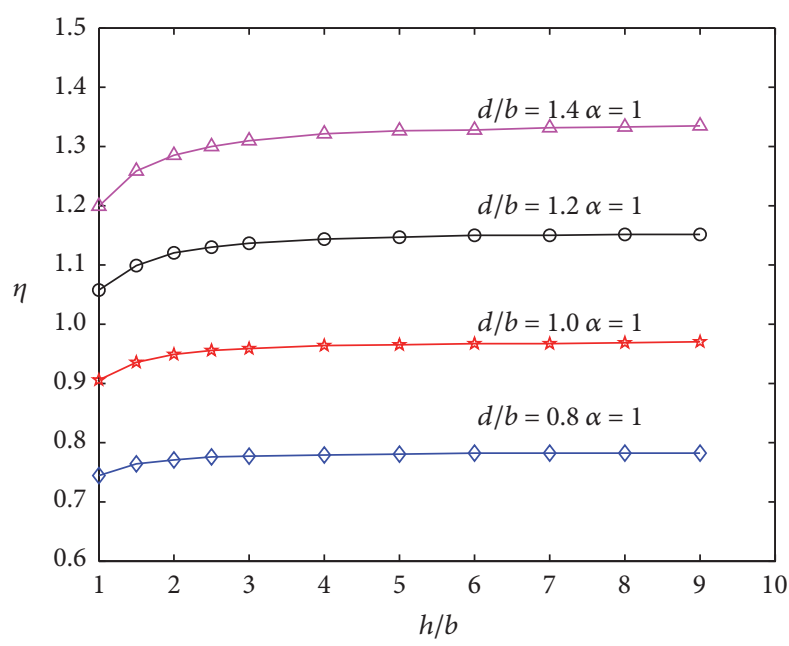

(a) Semi-infinite crystal

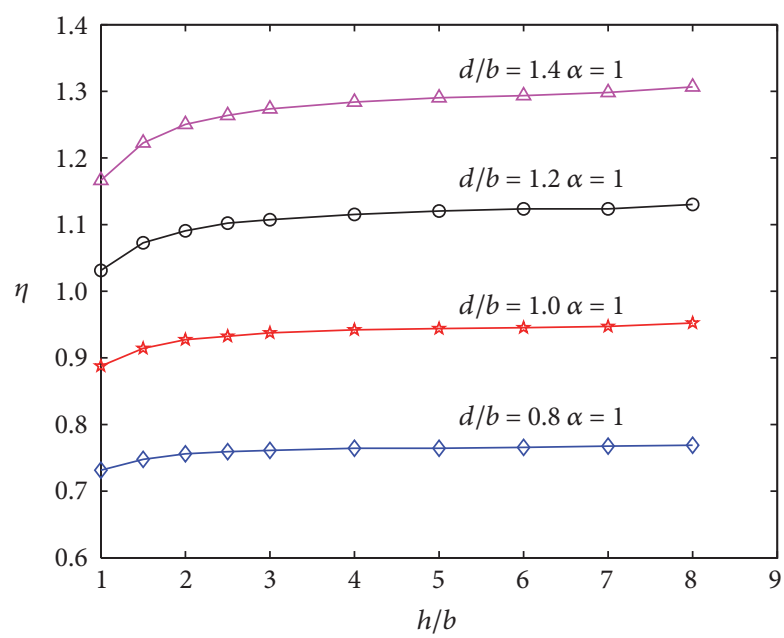

(c) $H / b=18$

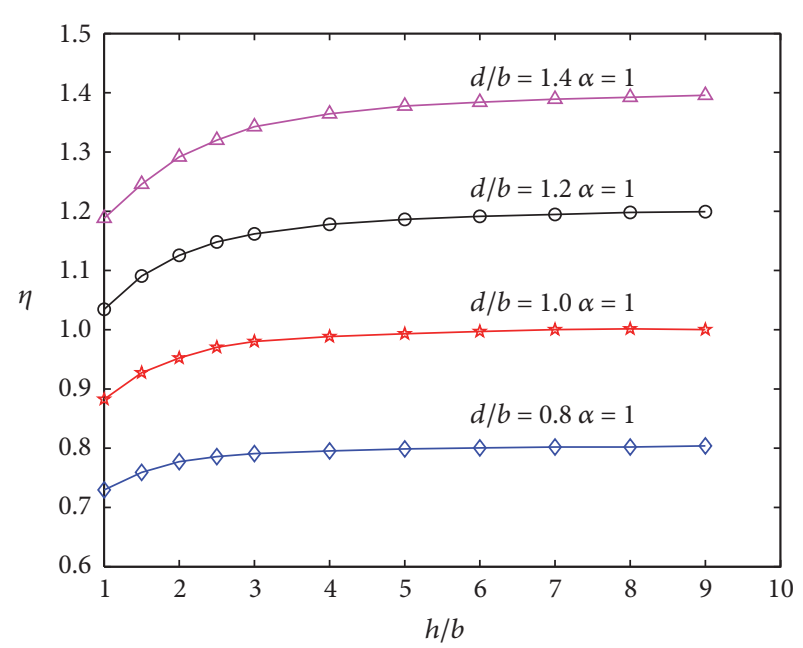

(b) $h=H / 2$

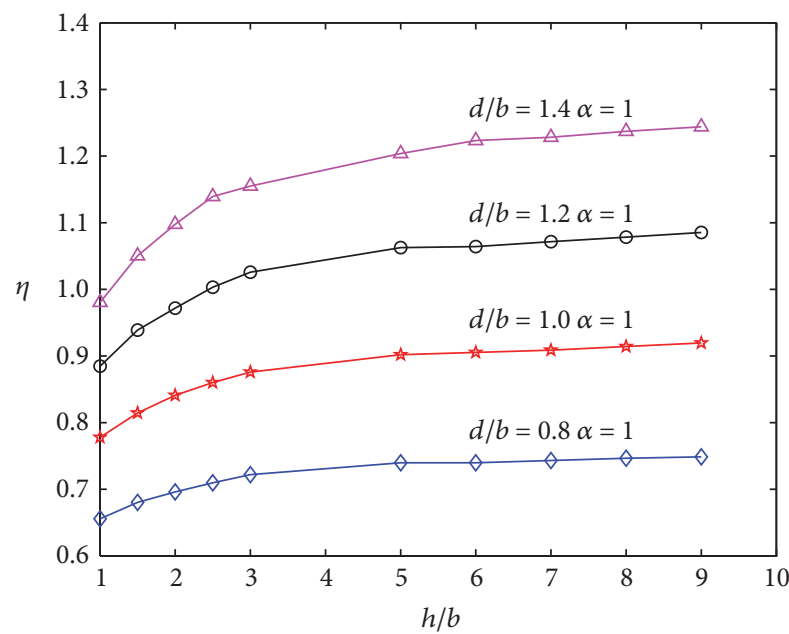

(d) $H / b=h / b+4$

FIGURE 3: Variation of core structure parameter $\eta$ with respect to the dislocation's distance to the surface for various interplanar atomistic spacing $(d / b)$ for screw dislocations.

shown in these figures, we can find that the amplification factor $\left(\sigma_{p} / \sigma_{p}^{0}\right)$ of screw dislocation decreases with respect to $h$ with the same $d / b$ and $\alpha$; it is shown that our results agree well with conclusion of Cheng et al. [10]. When we increase the interplanar atomistic spacing $(d / b)$, surprisingly contrary to what Cheng et al. concluded, it is found that $\sigma_{p} / \sigma_{p}^{0}$ increases, rather than decreasing with the same $h$ and $\alpha$ (see Figure 5). We believe that interplanar atomistic spacing $(d / b)$ has a better influence on Peierls stress than surface effect.

In this letter, we focus on the dislocation core structure and Peierls stresses of $(1 / 2)\langle 110\rangle\{111\}$ screw dislocations in $\mathrm{Al}$ and $\mathrm{Cu}$ nanofilms. The lattice constants and elastic constants are shown in Table 1 . The shear moduli $\mu$ of $\mathrm{Al}$ and $\mathrm{Cu}$ nanofilms can be determined from their elastic constants $C_{11}$, $C_{12}$, and $C_{44}$ via the equation $\mu=3 C_{44}\left(C_{11}-C_{12}\right) /\left(4 C_{44}+\right.$ $C_{11}+C_{12}$ ) [18]. The USF energies of $\mathrm{Al}$ and $\mathrm{Cu}$ nanofilms for different thickness are listed in Table 2. The USF energy
TABLE 1: The lattice constants $a$, Burgers vector $b$ and distance between slip planes $d$ (in units of $\AA$ ), elastic constants $C_{11}, C_{12}$, and $\mathrm{C}_{44}$ (in units of $\mathrm{GPa}$ ), and shear modulus $\mu$ of $\mathrm{Al}$ and $\mathrm{Cu}$.

\begin{tabular}{lccccccc}
\hline Materials & $a$ & $b$ & $d$ & $C_{11}$ & $C_{12}$ & $C_{44}$ & $\mu$ \\
\hline $\mathrm{Al}$ & 4.039 & 2.856 & 2.332 & 112.5 & 60.9 & 39.6 & 18.5 \\
$\mathrm{Cu}$ & 3.637 & 2.572 & 2.100 & 164.7 & 126.6 & 72.2 & 14.2 \\
\hline
\end{tabular}

deceases with the thickness of nanofilms, and the USF energies are the smallest for bulk $\mathrm{Al}$ and $\mathrm{Cu}$. This trend is in accordance with the results obtained by Datta et al. [19].

The core structure parameter is determined by substituting the dislocation solution equation (12) into the dislocation equation via comparing the maximum restoring force (see Figure 6). The dislocation densities are shown in Figure 7, and the core width of dislocations in nanofilms becomes 


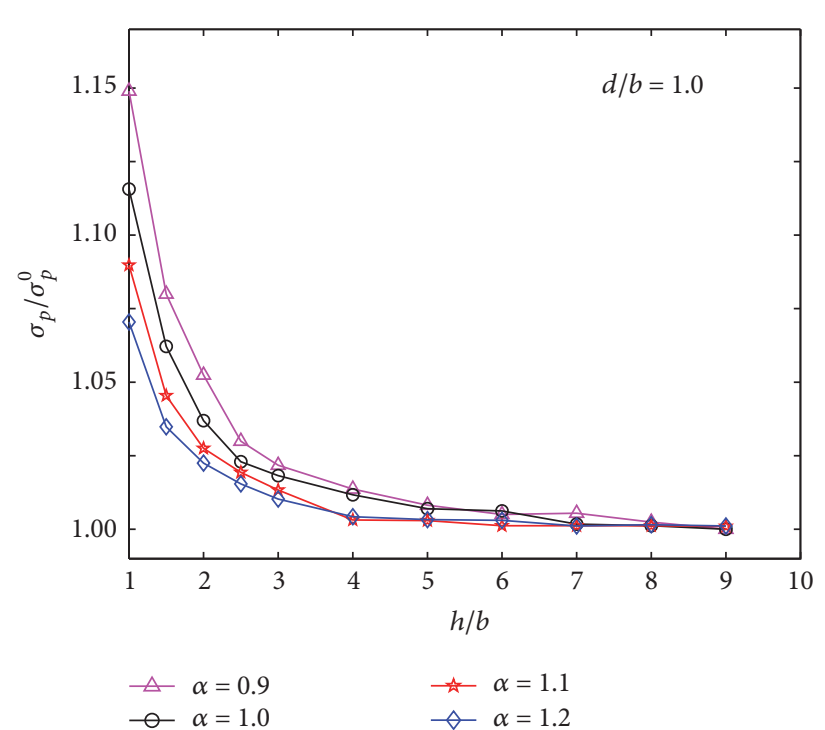

(a) Semi-infinite crystal

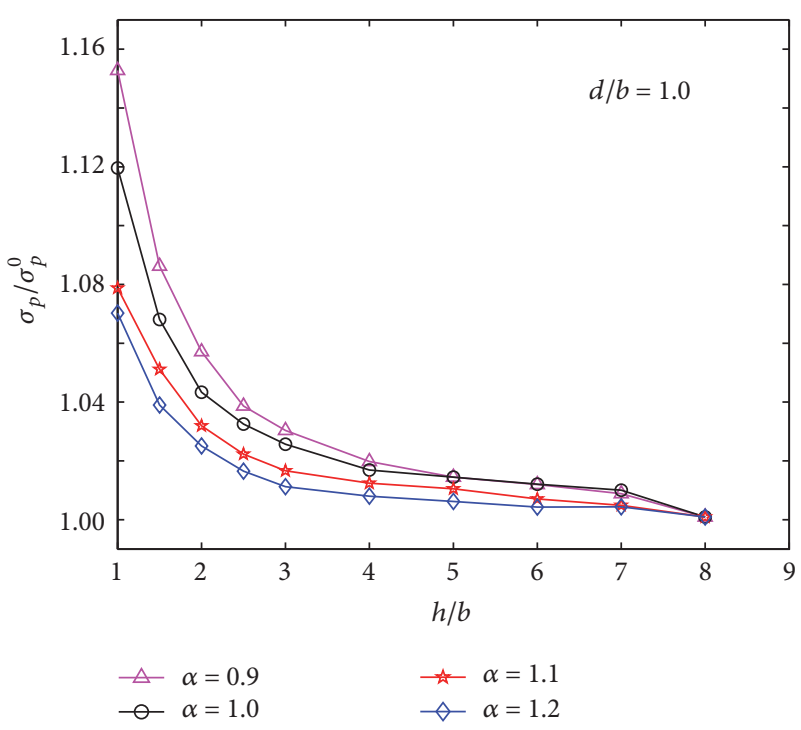

(c) $H / b=18$

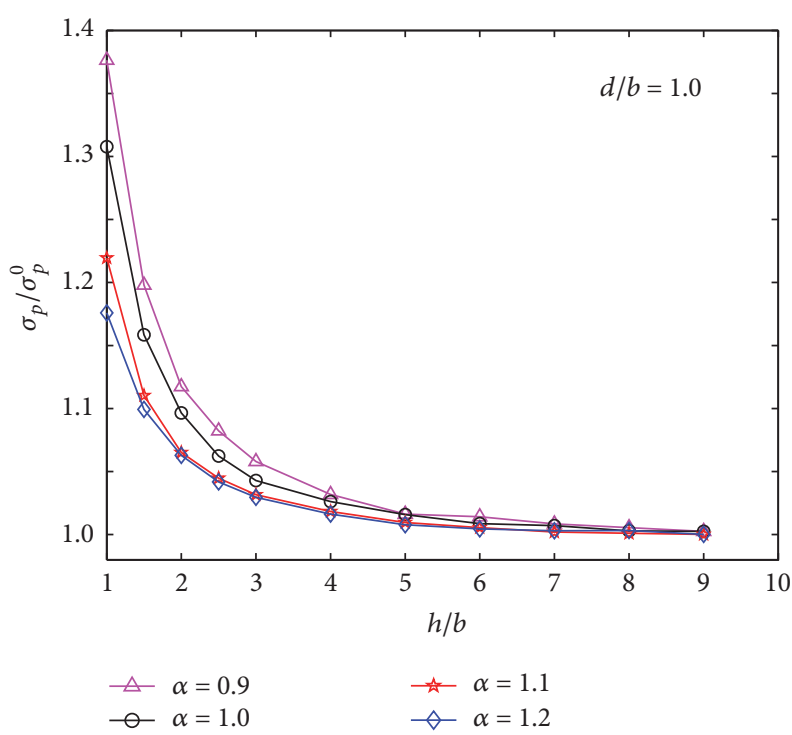

(b) $h=H / 2$

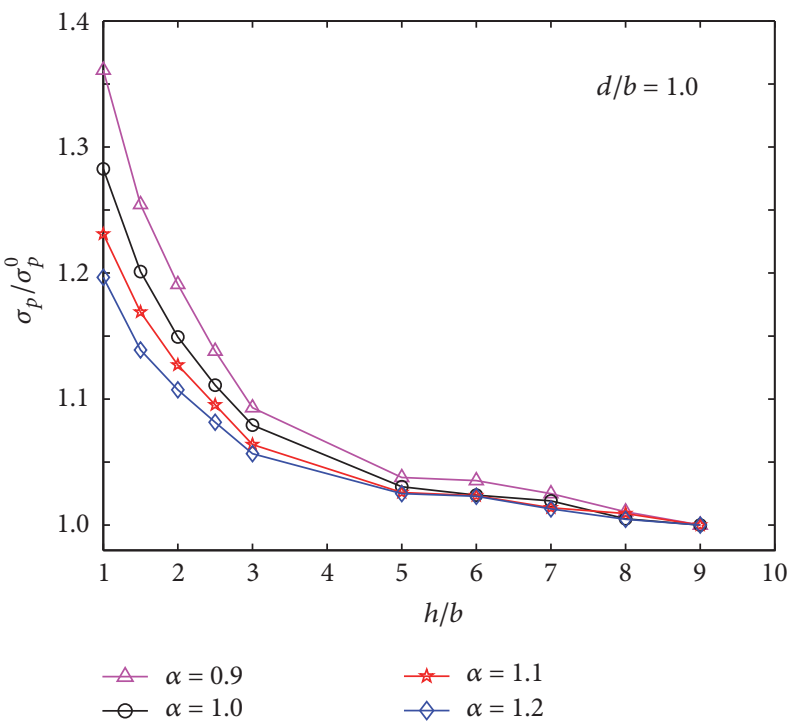

(d) $H / b=h / b+4$

Figure 4: Variation of the amplification factors of Peierls stress for a screw dislocation with respect to its distance to the surface for various normalized unstable stacking fault energy.

TABLE 2: The unstable stacking fault energy $\gamma_{\text {us }}$ (in units of $\mathrm{mJ} / \mathrm{m}^{2}$ ), core structure parameter $\eta$, core width $\xi$ (in units of $b$ ), and Peierls stress $\sigma_{p}$ (in units of $\mu$ ) of the $(1 / 2)\langle 110\rangle\{111\}$ screw dislocations in $\mathrm{Al}$ and $\mathrm{Cu}$ nanofilms.

\begin{tabular}{lcccccccc}
\hline \multirow{2}{*}{ Nanofilms } & \multicolumn{4}{c}{$\mathrm{Al}$} & \multicolumn{4}{c}{$\mathrm{Cu}$} \\
& $\gamma_{\text {us }}$ & $\eta$ & $\xi$ & $\sigma_{p}$ & $\gamma_{\text {us }}$ & $\eta$ & $\xi$ & $\sigma_{p}$ \\
\hline $3 \mid 6$ & 379.8 & 0.64 & 0.644 & 0.172 & 503.7 & 0.350 & 0.351 & 6.295 \\
$3 \mid 9$ & 356.2 & 0.69 & 0.693 & 0.147 & 498.4 & 0.357 & 0.357 & 6.109 \\
$6 \mid 9$ & 343.7 & 0.73 & 0.721 & 0.131 & 498.1 & 0.361 & 0.361 & 6.033 \\
Bulk & 339.4 & 0.79 & 0.792 & 0.118 & 495.7 & 0.371 & 0.391 & 5.850 \\
\hline
\end{tabular}

narrower than that in bulk (see Table 2 and Figure 7). Peierls stress decreases with the thickness of nanofilms.
Weinberger simulated the Peierls stress of an edge dislocation in molybdenum with modified Finnis-Sinclair potential to check the thickness dependence of the Peierls stress [11]. It is found that Peierls stress rises with decreasing thickness when thickness is below $8 \mathrm{~nm}$, and Peierls stress for large thicknesses converges to around $20 \mathrm{MPa}$ (Peierls stress of dislocations in bulk Mo). Our results are in agreement with the conclusions of Weinberger [11]. However, Lee and Li show that Peierls stress of a surface screw dislocation may be about $5-15 \%$ less than that in bulk materials based on a half-space P-N model [8]. Gars and Markenscoff accounted the effect of free surface on the Peierls stress by using the variable-core dislocation model [6]. The trend is the same as obtained by Lee and $\mathrm{Li}$, but the order of magnitude is different, due to the surface effects on the variable width. The opposite results 


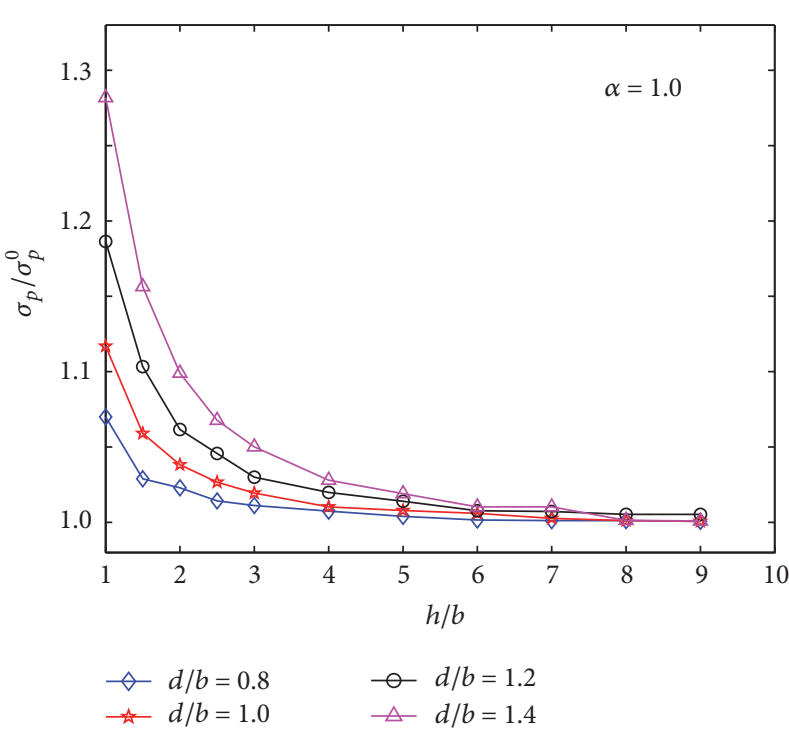

(a) Semi-infinite crystal

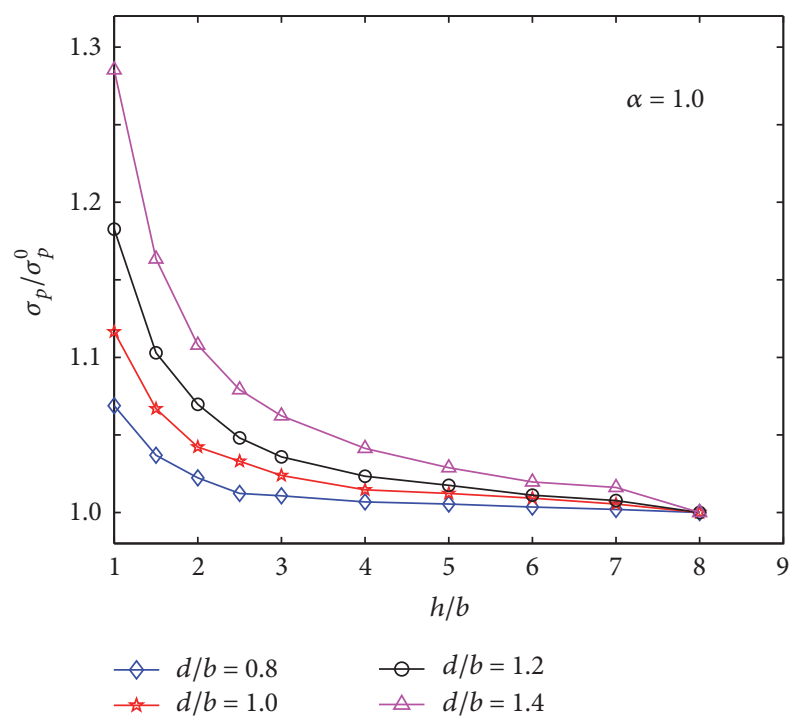

(c) $H / b=18$

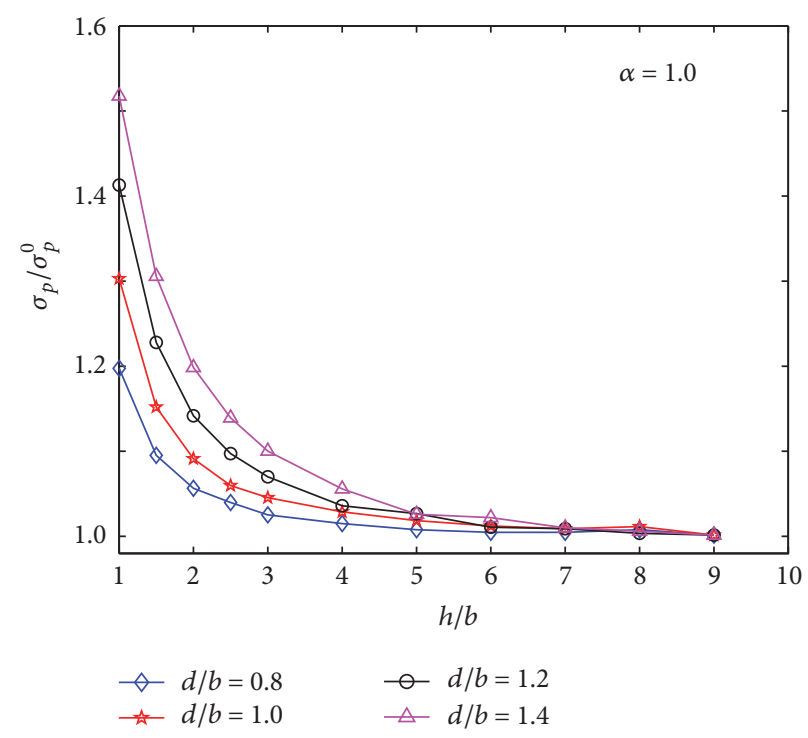

(b) $h=H / 2$

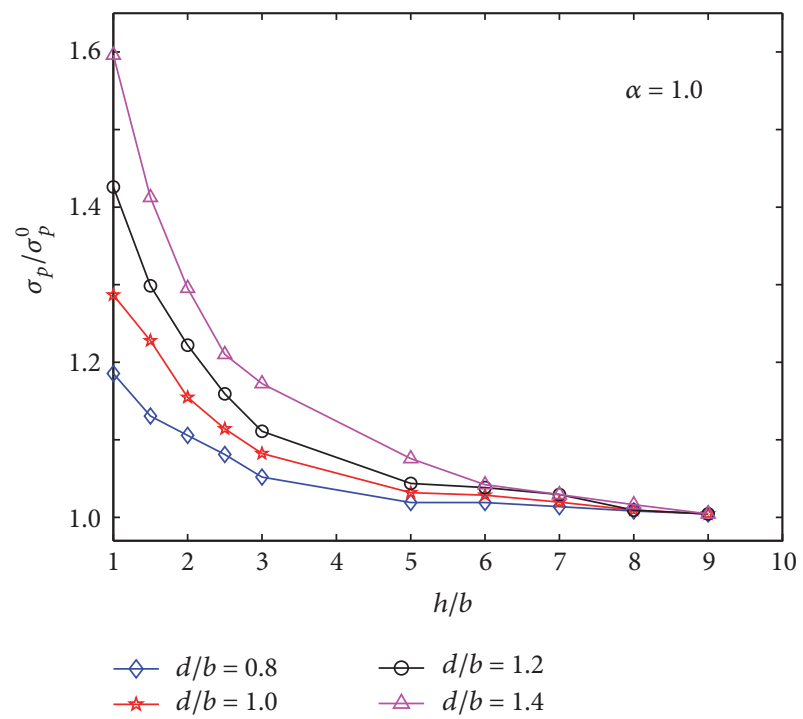

(d) $H / b=h / b+4$

Figure 5: Variation of the amplification factors of Peierls stress for a screw dislocation with respect to its distance to the surface for various interplanar atomistic spacing $(d / b)$.

originated from the missing change of dislocation profile under the free surface, as pointed out by Cheng et al. [10]. This shortage can be covered by the new dislocation solution presented here for dislocations in nanofilms.

\section{Conclusions}

In summary, we have constructed the governing equation of screw dislocations in nanofilms using the image dislocation method. The classical Peierls-Nabarro equation can be recovered when the thickness of nanofilm is taken to be infinite. We have studied the effects of USF energies, the spacing between the dislocation and the surface $(h)$, and the interplanar atomic spacing $(d / b)$ on both Peierls stress and core structure parameter, respectively. The core structure and Peierls stress of dislocations in $\mathrm{Al}$ and $\mathrm{Cu}$ nanofilms are determined by the governing equation combined with the USF energies obtained by the first-principle methods. It is found that surface effect can increase the Peierls stresses of screw dislocations in $\mathrm{Al}$ and $\mathrm{Cu}$ nanofilms.

\section{Competing Interests}

The authors declare that they have no competing interests.

\section{Acknowledgments}

The work is supported by the Scientific and Technological Research Program of Chongqing Municipal Education 


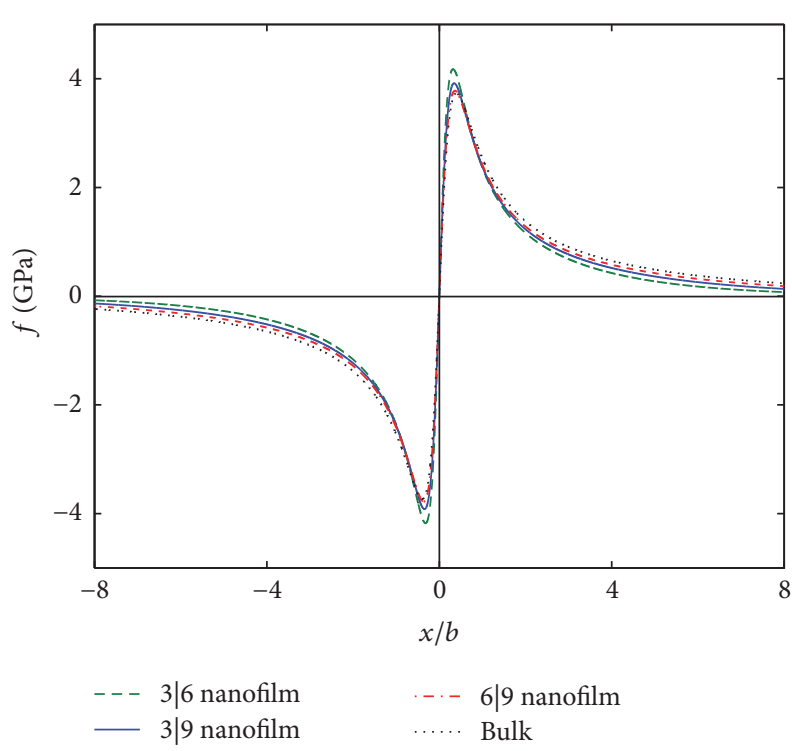

(a) $\mathrm{Al}$

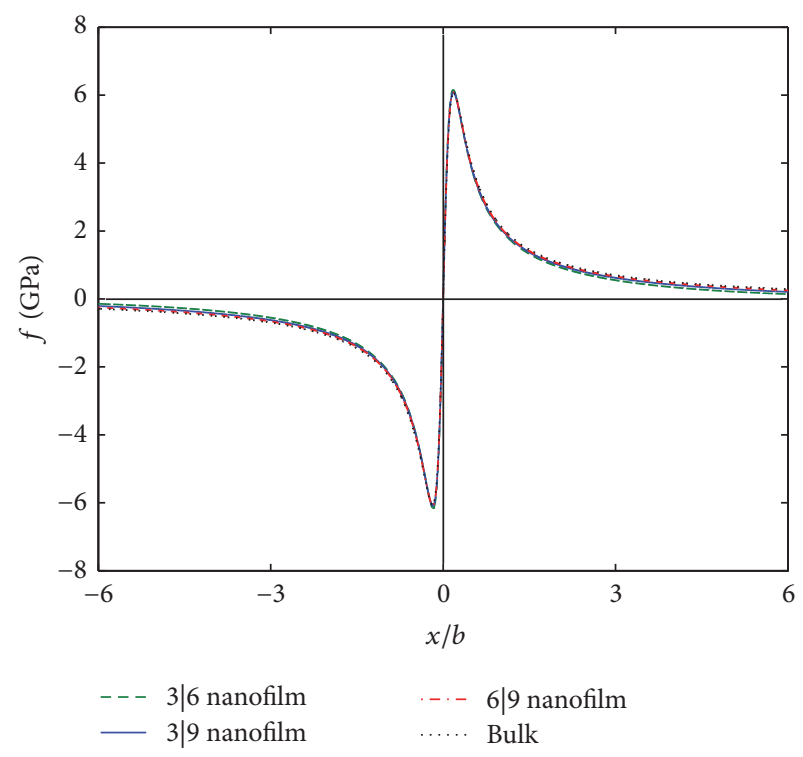

(b) $\mathrm{Cu}$

Figure 6: Stress fields of the $(1 / 2)\langle 110\rangle\{111\}$ screw dislocations in $\mathrm{Al}$ and Cu nanofilms for different $h \mid(H-h)$.

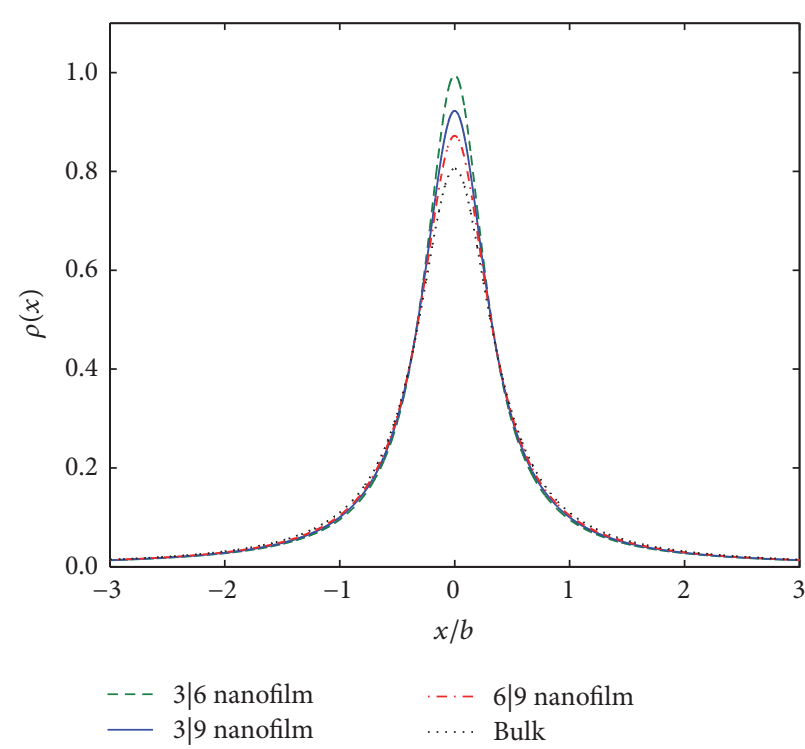

(a) $\mathrm{Al}$

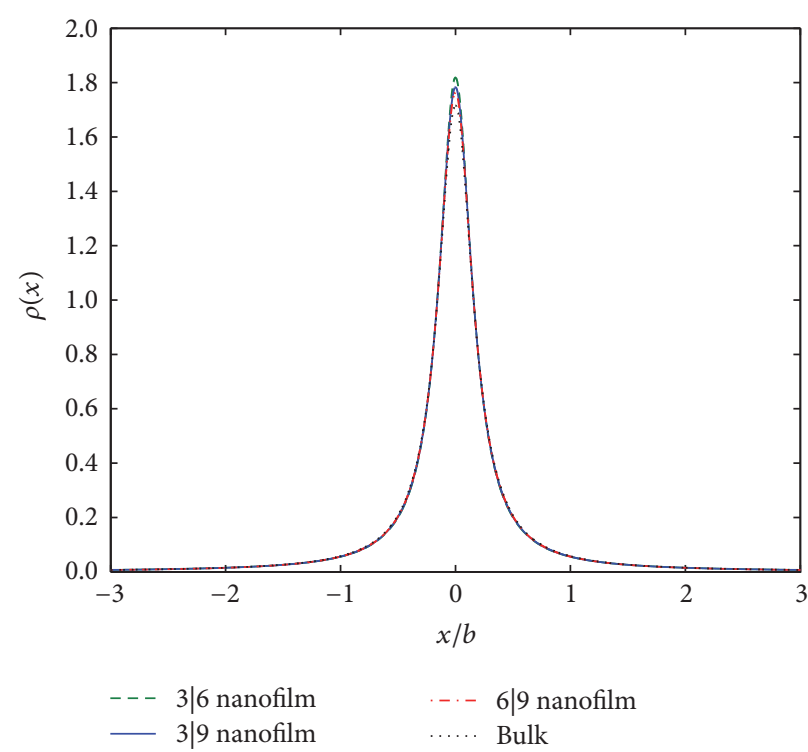

(b) $\mathrm{Cu}$

FIgURE 7: Dislocation densities of the $(1 / 2)\langle 110\rangle\{111\}$ screw dislocations in $\mathrm{Al}$ and $\mathrm{Cu}$ nanofilms for different $h \mid(H-h)$.

Commission (KJ1710252), the Natural Science Foundation of China (11104361), project supported by Program for Innovation Team Building at Institutions of Higher Education in Chongqing (CXTDX201601034), and the Frontier and Applied Basic Research Project of Chongqing, China (nos. cstc2014jcyjA1302 and cstc2016jcyjA1233).

\section{References}

[1] J. P. Hirth and J. Lothe, Theory of Dislocations, John Wiley \& Sons, New York, NY, USA, 2nd edition, 1982.
[2] R. Peierls, "The size of a dislocation," Proceedings of the Physical Society, vol. 52, no. 1, pp. 34-37, 1940.

[3] F. R. N. Nabarro, "Dislocations in a simple cubic lattice," Proceedings of the Physical Society of London, vol. 59, no. 2, article 256, 1947.

[4] D. Ferré, P. Carrez, and P. Cordier, "Modeling dislocation cores in $\mathrm{Sr} \mathrm{Ti} \mathrm{O}_{3}$ using the Peierls-Nabarro model," Physical Review B, vol. 77, no. 1, Article ID 014106, 2008.

[5] T. Fan, L. Luo, L. Ma, B. Tang, L. Peng, and W. Ding, "Effects of $\mathrm{Zn}$ atoms on the basal dislocation in magnesium solution from Peierls-Nabarro model," Materials Science and Engineering: A, vol. 582, pp. 299-304, 2013. 
[6] B. Gars and X. Markenscoff, "The Peierls stress for coupled dislocation partials near a free surface," Philosophical Magazine, vol. 92, no. 11, pp. 1390-1421, 2012.

[7] W. Wu, R. Schäublin, and J. Chen, "General dislocation image stress of anisotropic cubic thin film," Journal of Applied Physics, vol. 112, no. 9, p. 093522, 2012.

[8] C. Lee and S. Li, "A half-space Peierls-Nabarro model and the mobility of screw dislocations in a thin film," Acta Materialia, vol. 55, no. 6, pp. 2149-2157, 2007.

[9] C.-L. Lee and S. Li, "The size effect of thin films on the Peierls stress of edge dislocations," Mathematics and Mechanics of Solids, vol. 13, no. 3-4, pp. 316-335, 2008.

[10] X. Cheng, Y. Shen, L. Zhang, and X. H. Liu, "Surface effect on the screw dislocation mobility over the Peierls barrier," Philosophical Magazine Letters, vol. 92, no. 6, pp. 270-277, 2012.

[11] C. R. Weinberger, "Comment on 'Lattice Resistance to Dislocation Motion at the Nanoscale," Physical Review Letters, vol. 105, Article ID 099601, 2010.

[12] C. R. Weinberger, "Dislocation drag at the nanoscale," Acta Materialia, vol. 58, no. 19, pp. 6535-6541, 2010.

[13] K. Jagannadham and M. J. Marcinkowski, "Comparison of the image and surface dislocation models," Physica Status Solidi A, vol. 50, no. 1, pp. 293-302, 1978.

[14] B. Joós, Q. Ren, and M. S. Duesbery, "Peierls-Nabarro model of dislocations in silicon with generalized stacking-fault restoring forces," Physical Review B, vol. 50, no. 9, pp. 5890-5898, 1994.

[15] Y. Yao, T. Wang, and C. Wang, "Peierls-Nabarro model of interfacial misfit dislocation: an analytic solution," Physical Review B, vol. 59, no. 12, pp. 8232-8236, 1999.

[16] J. W. Christan and V. Vitek, "Dislocations and stacking faults," Reports on Progress in Physics, vol. 33, no. 1, p. 307, 1970.

[17] J. R. Rice, "Dislocation nucleation from a crack tip: an analysis based on the Peierls concept," Journal of the Mechanics and Physics of Solids, vol. 40, no. 2, pp. 239-271, 1992.

[18] D. Roundy, C. R. Krenn, M. L. Cohen, and J. W. Morris Jr., "Ideal shear strengths of fcc aluminum and copper," Physical Review Letters, vol. 82, no. 13, pp. 2713-2716, 1999.

[19] A. Datta, A. Srirangarajan, U. V. Waghmare, U. Ramamurty, and A. C. To, "Surface effects on stacking fault and twin formation in fcc nanofilms: a first-principles study," Computational Materials Science, vol. 50, no. 12, pp. 3342-3345, 2011. 

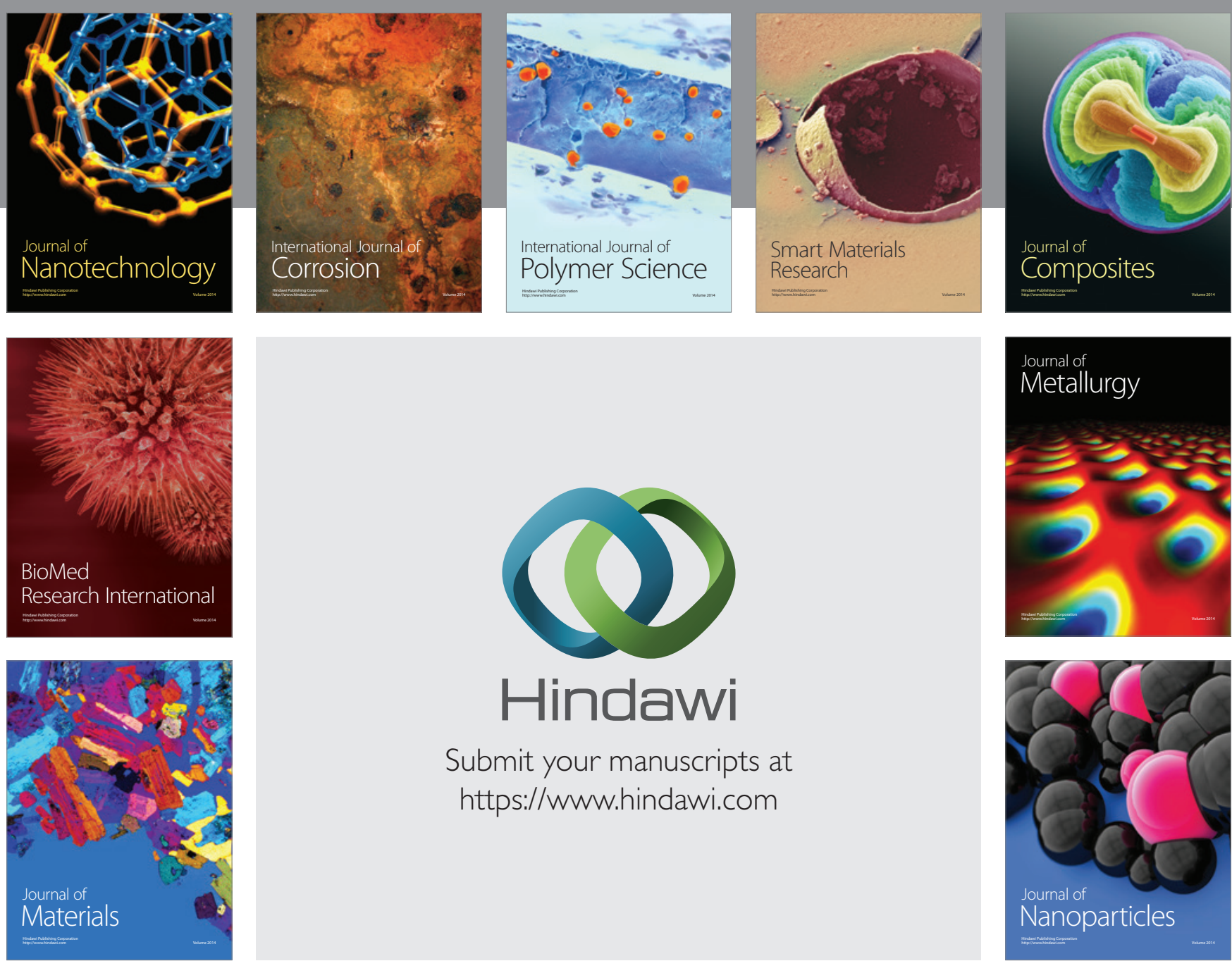

\section{Hindawi}

Submit your manuscripts at

https://www.hindawi.com

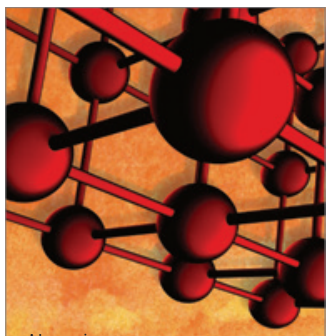

Materials Science and Engineering
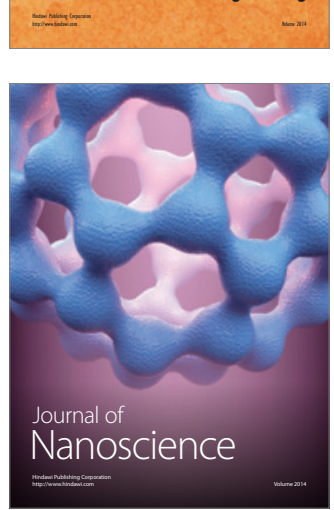
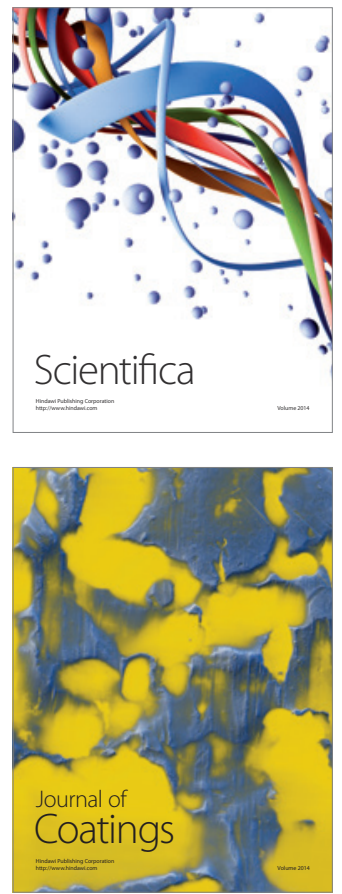
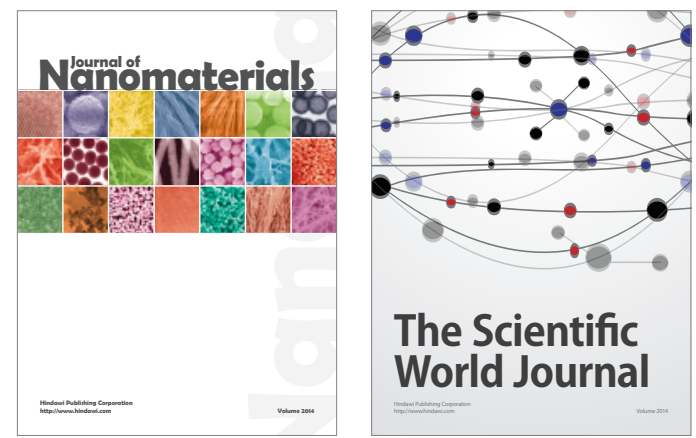

The Scientific World Journal
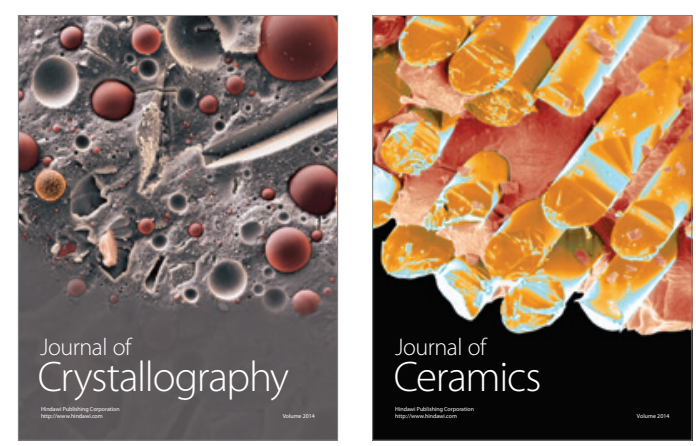
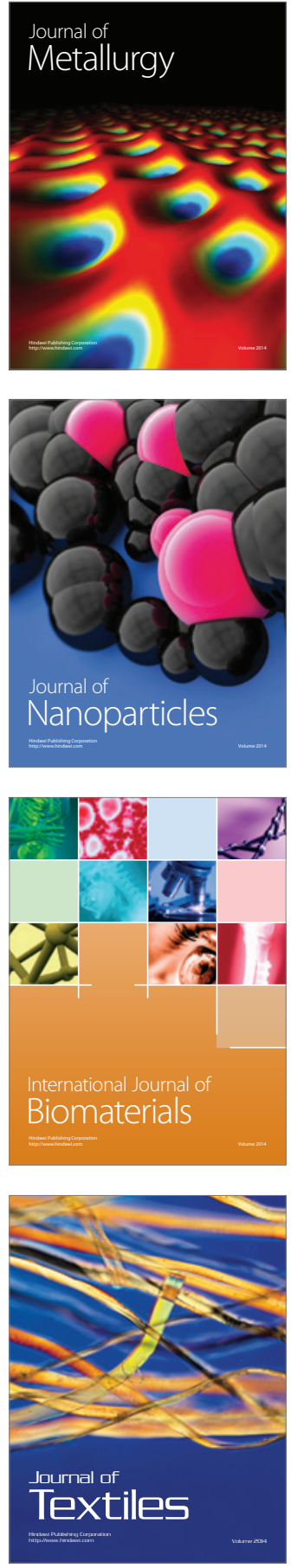